\title{
THE EFFECT OF FREQUENCY CHARACTERISTICS AND DURATION OF INPUT EARTHQUAKE MOTION ON THE ENERGY RESPONSE OF STRUCTURES
}

\author{
By Kiyoshi HIRAO*, Tsutomu SAWADA**, Yoshifumi NARIYUKI*** \\ and Syuji SASADA****
}

\begin{abstract}
The purpose of this study is to investigate the effect of frequency characteristics and duration of input earthquake motion on the energy responses of structures. In this study, numerical analysis for bi-linear SDOF structures with wide ranging structural parameters was carried out by the use of twelve artificial earthquakes, each varying in duration and frequency characteristics. From the examination of analytical results, it is found that the frequency characteristics of the input earthquake motion has a large effect on the periodical characteristics of all responses of the structures, such as elastic maximum velocity $\left(V_{\text {emax }}\right)$, elastic input energy $\left(E_{t e}\right)$, hysteretic energy $\left(E_{h \rho}\right)$ and so on. It is also found that the duration considerably affects the value of energy responses such as $E_{l e}$ and $E_{\text {ho. }}$

Keywoords : seismic damage, energy response, SDOF structures
\end{abstract}

\section{INTRODUCTION}

Recently, the idea of the two step earthquake resistant design has been taking root. The fundamental concepts behind this design include the following; (1) structures should be prevented from collapsing during the most severe earthquakes possible and (2) structures should remain within the elastic range for earthquakes expected to occur several times during the lifetime of a structure. In connection with this idea, the inelastic responses, especially, the energy responses of the structures excited by severe earthquakes has attracted special interest, and many studies on the input energy and the hysteretic energy/plastic strain energy have been carried out ${ }^{1)-5}$. From these studies, it is clear that input energy and hysteretic energy are among the best parameters to evaluate the seismic force of earthquakes and to measure the damage of structures, respectively. Moreover the qualitative and quantitative relation between these energies and structural parameters, such as the damping factor, yield strength and so on, have become rather clear.

However, it is difficult to clarify the relation between inelastic responses/energy responses for structures and characteristics of input earthquake motions, because the earthquake itself is a phenomenon with a lot of variations in frequency characteristics and duration, these factors having a large effect on the periodical characteristics and value of the responses of structures, respectively. In relation to this, Ohno et al. ${ }^{6}$ reported that the ratio of the hysteretic energy to the input energy is scarcely affected by the difference of input earthquake motions, because the effect of the earthquake motion on these energies is

* Member of JSCE, Dr. of Eng., Professor, Dept. of Civil Eng., University of Tokushima (Minamijosanjima 2-1, Tokushima, 770, Japan)

** Member of JSCE, Lecturer, Dept. of Construction Eng., University of Tokushima (ditto)

*** Member of JSCE, Lecturer, Dept. of Civil Eng., University of Tokushima (ditto)

**** Member of JSCE, Assistant, Dept. of Civil Eng., Anan College of Technology (Aoki 265, Anan, 774, Japan) 
canceled out. Hakuno et al. ${ }^{7)}$, Zahrah et al. ${ }^{8)}$ and Matsushima et al. ${ }^{9)}$ reported that the plastic maximum displacement ${ }^{7)}$, the input energy ${ }^{8)}$ and the cumulative plastic deformation ratio $0^{9)}$ becomes larger as the duration increases. Matsushima et al. ${ }^{9)}$ also reported that the change in the cumulative deformation ratio due to the difference of the input earthquake motions is small, when the total power of each earthquake is the same. Thus, from these reports, the qualitative effect of input earthquake motion on the inelastic responses is gradually becoming clear. But most of these results are based on the information obtained by limited input earthquake motions and structures. Therefore, more wide ranging and systematic investigations on this qualitative effect are necessary. Also, when considering aseismic design of actual structure, it is necessary to grasp the quantitative effect of the input earthquake motions on the hysteretic energy, that is, the damage of the structures.

In order to make these points clear, in this study, numerical analysis for bi-linear SDOF structures with wide ranging structural parameters was carried out by the use of artificial earthquakes, each varying in frequency characteristics and duration. On the basis of these analytical results, the authors examined the quantitative and qualitative effect of frequency characteristics and duration of the input earthquake motions on the elastic maximum velocity $V_{e \max }$, the elastic input energy $E_{i e}$ and the hysteretic energy $E_{h p}$. In order to maintain the generality of the analytical results, the artificial earthquakes were generated such that their acceleration response factor spectra agree with the target ones, each varying in magnitude, epicentral distance and ground condition, as shown in the Japanese specifications for road bridges $\mathrm{V}^{10)}$.

\section{INPUT EARTHQUAKE MOTION}

As was stated previously in this study, in order to maintain the generality of the analytical results, we used the average acceleration response factor spectra shown in the Japanese specifications for road bridges $\mathrm{V}^{10)}$; the artificial earthquakes Nos. 1 to 12 were generated by the method used in Ref. (11). Table 1 shows the magnitude $M$, epicentral distance $D$ and ground condition $G C$, targeted in each artificial earthquake. It also shows the total duration $T_{t}$, the duration of the strong motion $T_{s}$, the average time corresponding to maximum value of strong motion part $t_{m}$ and the total power $P_{t}$ in each earthquake. The symbols $[\mathrm{A}],[\mathrm{B}]$ and $[\mathrm{C}]$ in Table 1 finds meaning within the scope of the following values ;

[A] : $M=5.4-6.4, D=0-19 \mathrm{~km}$ (near site and small scale earthquake)

[B] : $M=6.8-7.4, D=60-119 \mathrm{~km}$ (mid site and middle scale earthquake)

[C] : $M=7.5-7.9, \quad D=120-199 \mathrm{~km}$ (far site and large scale earthquake)

The duration $T_{s}$ of the earthquakes Nos. $1,3,5,7,9$ and 11 was calculated according to the $M-D$ regression equation in Ref. (12). As for the other earthquakes Nos. 2, 4, 6, 8, 10 and 12, the value of one half of each $T_{s}$ of Nos. 1, 3, 5, 7, 9 and 11 was used as shown in Table 1, to investigate the effect of the duration $T_{s}$ on the energy response. Similarly, as for the average time $t_{m}$, one third of the total duration $T_{t}$ was used in each earthquake.

Table 1 Earthquake data.

\begin{tabular}{|c|c|c|c|c|c|c|}
\hline $\begin{array}{c}\mathrm{F}, \mathrm{Q} \\
\text { number }\end{array}$ & $M \cdot D$ & $\mathrm{GC}$ & $\begin{array}{c}\mathrm{T}_{\mathrm{t}} \\
(\mathrm{sec})\end{array}$ & $\begin{array}{c}\mathrm{t} \cdot \mathrm{m} \\
(\mathrm{sec})\end{array}$ & $\begin{array}{c}T_{s} \\
(s e c)\end{array}$ & $\begin{array}{c}\mathrm{P}_{\mathrm{t}} \\
\left.(g a)^{2} \cdot \sec \right)\end{array}$ \\
\hline No. 1 & $\Lambda]$ & 3 & 40.96 & 13.65 & 3.66 & 86506 \\
\hline No. 2 & $\Lambda \mathrm{J}$ & 3 & $"$ & $"$ & 1.81 & \\
\hline No. 3 & $\bar{B}$ & 3 & 81.92 & 27.30 & 11.50 & \\
\hline No. 1 & $\bar{B}$ & 3 & 1 & 11 & 5.75 & \\
\hline No. 5 & $C$ & 3 & 163.84 & 51.61 & 17.10 & \\
\hline No. 6 & $C$ & 3 & II & " & 8.55 & \\
\hline No. 7 & $\bar{B}$ & 1 & 81.92 & 27.30 & 11.50 & \\
\hline No. 8 & $\bar{B}$ & $i$ & 11 & 11 & 5.75 & \\
\hline No. & $\bar{B}$ & 2 & II & "I & 11.50 & \\
\hline No. 10 & $\mathrm{~B}$ & 2 & $"$ & $"$ & 5.75 & \\
\hline No. 1 & B & 4 & $" 1$ & $" 1$ & 11.50 & \\
\hline No. 12 & B & 4 & $" 1$ & $" 1$ & 5.75 & 118274 \\
\hline \multicolumn{7}{|c|}{$\begin{array}{l}(M \cdot D)=[A]: M=5.1 \sim 6.4 \text {, and } D=0 \sim 19 \mathrm{~km}, \quad P \text { total power } \\
(M \cdot D)=[B]: M=6.8 \sim 7.4 \text {, and } D=60 \sim 119 \mathrm{~km}, \quad G C=\text { Ground Condition } \\
(M \cdot D)=[C]: M=7.5 \sim 7.9 \text {, and } D=120 \sim 199 \mathrm{~km}, T_{t}=\text { total duration } \\
t . \\
=\text { the mean time of strong motion, } T_{s}=\text { duration of strong motion }\end{array}$} \\
\hline
\end{tabular}
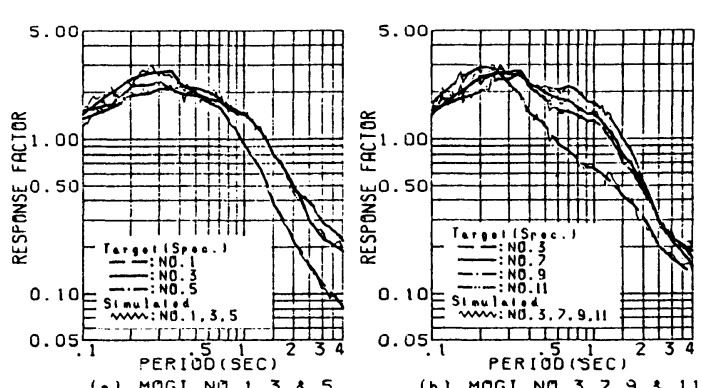

Fig. 1 Target and simulated acceleration response factor spectra. 
Figs. 1 (a) and ( b ) show the simulated response factor spectra together with the target ones for earthquakes Nos. 1, 3, 5 and Nos. 3, 7, 9, 11, respectively. In this case, Nos. 1, 3 and 5 are earthquakes which have the same ground condition of $G C=3$ and different combinations for magnitude and epicentral distance of $(M \cdot D)=[\mathrm{A}],[\mathrm{B}]$ and $[\mathrm{C}]$, respectively. Nos. 3, 7, 9 and 11 are the same $(M \cdot D)$ as $(M \cdot D)=[\mathrm{B}]$ and different $G C^{\prime}$ s, i. e., $G C=3, G C=1, G C=2$ and $G C=4$. From this figure, it is noted that the results of the simulated spectra are very similar to the target ones.

The acceleration wave forms, normalized by 300 gals in maximum acceleration, for Nos. $1,3,5$ and Nos. 7, 9, 11 and their respective Fourier spectra are shown in Figs. 2 and 3, in which, the reduced scale in time axis differs in each of Fig. 2.

From examining Table 1 and Fig. 1 and Figs. 2 and 3, the following points become clear;

(1) For the acceleration wave forms of the simulated earthquakes used in this study, the average time $t_{m}$ and the spread of the strong motion differ according to the value of the total duration $T_{t}$ and the duration of the strong motion $T_{s}$. With the increasing values of $T_{t}$ and $T_{s}$, the duration of the wave gets proportionally larger, although, the form of each wave is almost similar.

(2) There is a close relation between the frequency/periodical characteristics in the acceleration response factor spectrum and those in the Fourier spectrum, for any simulated earthquake (see Figs. 1, 2 and 3). The difference of the frequency characteristics in the earthquakes depends on the magnitude and epicentral distance $(M \cdot D)$ and the ground condition $G C$. That is, when the ground condition is the same as $G C=3$ (see Fig. 2), the spectrum value of earthquake No. 1 with small $(M \cdot D)$ becomes rather smaller compared with those in Nos. 3 and 5, in the range of the natural period less than 1 sec.. Also in a comparison of spectrum values between No. 3 and No. 5 , the former maintains larger values with a period range less than $0.4 \mathrm{sec}$. . There is also a tendency for these values to be a little smaller in the range of the period longer than 1 or 2 sec. . On the other hand, when $(M \cdot D)$ is the same as $(M \cdot D)=[B]$ and $G C$ differs (see Fig. 3), the value of the spectra shows a tendency to become larger in the range of the period longer than $0.3 \mathrm{sec}$, and smaller in the range shorter than $0.3 \mathrm{sec}$, as the ground gradually becomes softer from No.7 onwards, e.g. No.7 $(G C=1)$, No. $9(G C=2)$, No. $11(G C=4)$. This tendency is remarkable between No. 7 for the type 1 ground and others. However the No. 9 Fourier spectrum for the type 2 ground is very similar to the one of No. 3 for the type 3 ground. The figures were omitted, for earthquakes Nos. 2, 4, 6, 8, 10 and 12 , as the individual effect of $(M \cdot D)$ and $G C$ on their Fourier spectra is almost the same as the results mentioned above.
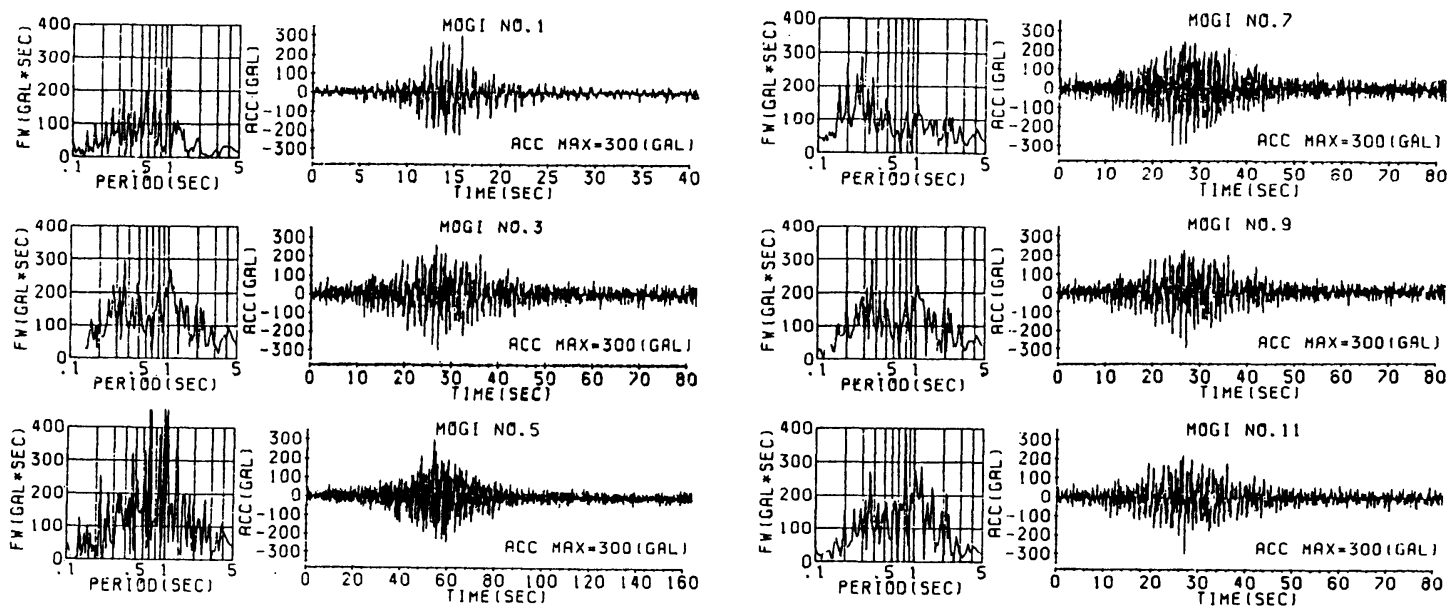

Fig. 2 Accelerograms and Fourier spectra of Nos. 1, 3, 5 $(G C=3,(M \cdot D)=[\mathrm{A}],[\mathrm{B}],[\mathrm{C}])$.

Fig. 3 Accelerograms and Fourier spectra of Nos.7, 9, 11 $(M \cdot D)=[\mathrm{B}], G C=1,2,4)$. 
(3) A near proportional relation is found between the duration $T_{s}$ and the total power $P_{t}$ for each earthquake (see Table 1) - namely, under the same ground as $G C=3, P_{t}$ becomes larger as $T_{s}$ gets longer due to the increase of $(M \cdot D)$; under the same $(M \cdot D)$ and $G C$ factors, $P_{t}$ is reduced by about one half as $T_{s}$ becomes one half. On the other hand, when $T_{s}$ is the same, even under the different frequency characteristics due to the change of ground condition, $P_{t}$ does not show much change. From these results, it seems that the total power $P_{t}$ is a reliable index to represent the duration of the strong motion $T_{s}$ in every earthquake.

\section{STRUCTURAL CHARACTERISTICS AND ANALYTICAL METHOD}

In this study, a bi-linear model as shown in Fig. 4 was used to demonstrate the characteristics of a restoring force for SDOF structures. In this model, as for the secondary slope $p$ which represents the ratio of plastic stiffness to elastic stiffness, the five values, $0.0,0.125$, $0.25,0.375$ and 0.5 , were selected. The yield strength ratio $R$ defined by formula ( 1 ) was used instead of $Q_{y}$ (see Ref. (5)) : Three values of $R, 0.25,0.5$ and 0.75 were also selected to correspond to each case where the inelastic response is strong, intermediate and weak, respectively.

$$
R=Q_{y} / Q_{e \max }=X_{y} / X_{\text {emax }}
$$

in which $X_{y}$ is the yield displacement, $Q_{e \max }$ and $X_{\text {emax }}$ is the maximum restoring force and displacement obtained from the elastic response analysis.

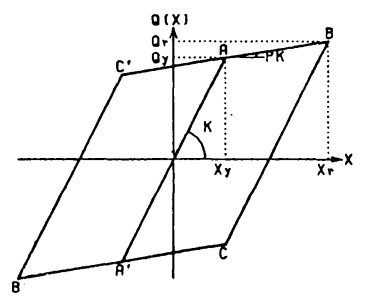

Fig. 4 Bi-linear model.

Considering the ordinary values of actual structures, the range for the damping factor $h=1$ to $10 \%$ and the natural period $T=0.1$ to $5 \mathrm{sec}$. was investigated. The following five values for $h$ were used, $1,2.5$, $5.0,7.5$ and $10 \%$. For $T$, after dividing the above range into nineteen equal parts on the logarithmic axis, twenty different values ${ }^{5)}$ were employed.

The analytical method used in this study is the same as in Ref. (5), so details of explanation are omitted. For the equation of motion and energy response of a SDOF structure excited by an earthquake, formulas (2) and (3), normalized by the mass $m$ and the yield displacement $X_{y}$ of the structure, were also employed. These formulas were integrated numerically by the linear acceleration method and the trapezoidal formula, respectively.

$$
\begin{aligned}
& \overline{\ddot{X}}+2 h w \dot{\bar{X}}+\omega^{2} \bar{Q}(\bar{X})=-\overline{\bar{X}}_{0} \ldots \ldots \ldots \ldots \ldots \ldots \ldots \ldots \ldots \ldots \ldots \ldots \ldots \ldots \ldots \ldots \ldots \\
& \int_{0}^{t} \ddot{\bar{X}} \cdot \dot{\bar{X}} d t+2 h w \int_{0}^{t} \dot{\bar{X}} \cdot \dot{\bar{X}} d t+\omega^{2} \int_{0}^{\bar{x}} \bar{Q}(\bar{X}) d \bar{X}=-\int_{0}^{t} \ddot{X}_{0} \cdot \dot{\bar{X}} d t
\end{aligned}
$$

where, $\bar{X}, \dot{\bar{X}}$ and $\ddot{\bar{X}}$ is the relative displacement, velocity and acceleration normalized by $X_{y}$, respectively. $h, \omega$ and $\bar{Q}(\bar{X})$ is the damping factor, natural circular frequency and normalized restoring force by $Q_{y}$, respectively, and $\ddot{\bar{X}}_{0}$ is the acceleration of input earthquake motion normalized by $X_{y}$.

As for the analytical results shown in the next section, the actual relative velocity obtained by multiplying $X_{y}$ to $\dot{\bar{X}}$ will be used, and the energy per unit mass obtained by multiplying $X_{y}^{2}$ to the result of formula (3) will also be used. SDOF structures were analyzed over the total duration $T_{t}$ of each earthquake as shown in Table 1, and equally spaced time interval of $d t=0.02 \mathrm{sec}$. was subdivided in the numerical integration, according to both the damping factor $h$ and natural period $T^{5)}$.

The third term of the left side in Eq. (3) / is the hysteretic energy which is absorbed into the structure/ and the right hand indicates the input energy which is inputted into the structure by the earthquake motion.

\section{ANALYTICAL RESULTS AND REMARKS}

In this study, for all combinations of the structural parameters and input earthquakes described in previous chapters, the numerical response analysis was carried out. However, only major examples of the 
results are shown. An attention is focused on the response of elastic maximum velocity $V_{\text {emax }}$, elastic input energy $E_{t e}$ and hysteretic energy $E_{h p}$, and the effect of the structural parameters, frequency characteristics and total power/duration on these responses is examined.

\section{(1) Effect of the structural parameters}

$V_{e \max }, E_{i e}$ and $E_{h p}$ against every natural period $T$ for the three values of the damping factor $h, 2.5,5.0$ and 7.5\%, are shown in Figs. 5 (a), (b) and (c) ; these are the results obtained for the earthquakes Nos. 1 and 3. For $E_{h p}$, four results of pairs of yield strength ratio $R$ with secondary slope $p(R=0.25, p=$ $0.0),(R=0.25, p=0.5),(R=0.5, p=0.0)$ and $(R=0.5, p=0.5)$ are shown, as can be seen in the upper part of each figure. In Fig. 5 ( $c)$, each result of the different input earthquakes and damping factors is distinguished by the thickness and type of the line as shown in each figure.

The figures show that every response is highly affected by the frequency characteristics of the input earthquake motions (see Fig. 2).

The effect of the damping factor $h$ is different among $V_{e \max }, E_{h p}$ and $E_{t e}$, that is, $E_{i e}$ is not affected so much by $h$. On the other hand, the effect of $h$ on $V_{e \max }$ and $E_{h \rho}$ is remarkable, for example, the values of these responses become smaller and their periodical characteristics are smoothed, as $h$ becomes larger. Hence, regarding the details of these values, discussion seems to be necessary concerning the value of $h$. But, as the comparison between thick lines and thin lines shows, there is not a great amount of change between earthquake No. 1 and No. 3, due to the value of $h$. For the sake of simplicity, only the case of $h=$ $5 \%$ will be discussed in the following.

Next, comparing the results of $E_{h \rho} p=0.0$ and $p=0.5$ (see Fig. $5(\mathrm{c})$ ), it can be seen that this $E_{h \rho}$ value is not affected very much by the secondary slope $p$. On the other hand, the effect of the yield strength ratio $R$ on $E_{h p}$ is significant, that is, the value of $E_{h p}$ gets larger as $R$ becomes smaller. Thus the ratio $R$ is thought to be one of the significant parameters in the discussion of the hysteretic energy $E_{h p}$. In the case of $R=0.75$, the comparison in the figure is difficult, because the value of $E_{h D}$ becomes smaller than those values in the case of $R=0.25$ and 0.5 . Therefore the result for $R=0.75$ has been omitted in this paper. Also as was mentioned above, the effect of the secondary slope $p$ is so small that only the results for $p=$ 0.0 will be given below.

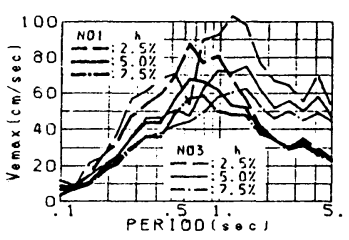

(a ) Vemax
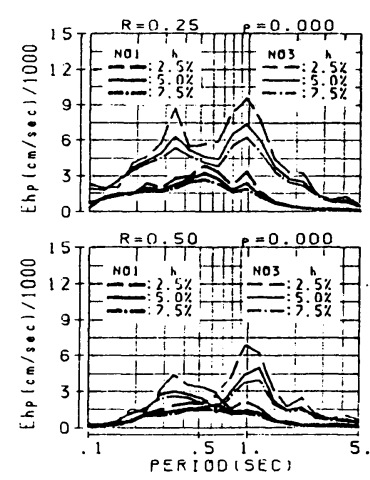

(c) Ehp

Fig. 5 Effect of structural parameters on $V_{e m a x}, E_{i e}$ and $E_{h p}$.

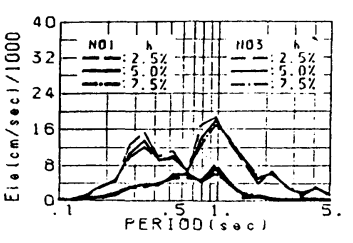

(b) Eie
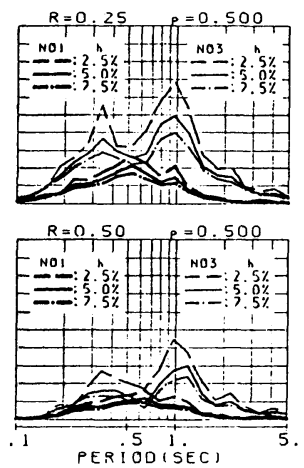

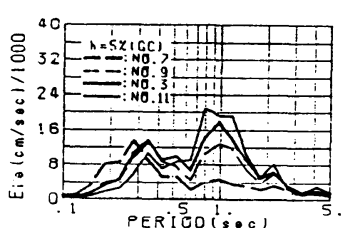

(a) Eie

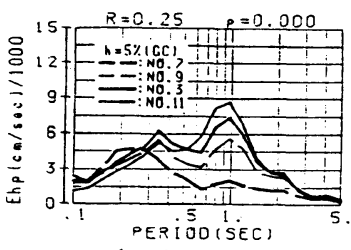

(b) Ehp

Fig. 6 Effect of ground condition $(G C)$ on $E_{i e}$ and $E_{h p}$. 
Figs. 6 (a) and (b) indicate the results of $E_{t e}$ and $E_{h p}$, respectively. In order to observe the difference of the ground condition $G C$, these results have been obtained from earthquakes No. $7(G C=1)$, No. $9(G C=2)$, No. $3(G C=3)$ and No. $11(G C=4)$ for the pair $(R=0.25, p=0.0)$ and the damping factor $h=5 \%$. Both the results of No. 9 for the type 2 ground and No. 3 for the type 3 ground are very similar, though the value of the latter becomes a little larger than that of the former. Therefore, in order to avoid complication in the figure, the results of the type 2 ground, that is, results of earthquakes Nos. 9 and 10 will be also omitted hereafter.

\section{(2) Effect of the frequency characteristics}

Fig. 7 shows the elastic maximum velocity $V_{e m a x}$, the elastic input energy $E_{l e}$ and the ratio of the equivalent velocity $\sqrt{2 E_{t e}}$ to $V_{e \max }$ for earthquakes Nos. 1 to 6 . Fig. 8 shows the same results for earthquakes Nos. 3, 4, 7, 8, 11 and 12. Fig. 7 represents the case in which the ground condition is the same as that of the type $3(G C=3)$ and the magnitude and epicentral distance $(M \cdot D)$, the total duration $T_{t}$ and the duration of the strong motion $T_{s}$ are different. In Fig. 8, $(M \cdot D)$ and $T_{t}$ are the same, whereas $G C$ and $T_{s}$ are different.

From Figs. 7 and 8, Figs. 2 and 3 and Table 1, the following points can be noted ;

(a) For each input earthquake motion, a good correlation can be found between the periodical characteristics of $V_{\text {emax }}$ and those of the Fourier spectrum. A similar relation can be also found in the relative between periodical characteristics of $V_{e \max }$ and those of the Fourier spectra, caused by the differences of $(M \cdot D)$ and $G C$ (see Figs. 7(a ), 8(a) and Figs. 2, 3). However, in the figure, as can be seen by comparing the thick and thin lines, there is no apparent tendency due to the difference of the duration of the strong motion $T_{s}$.

(b) A strong similarity can be seen between the periodical characteristics of $E_{t e}$ and those of the Fourier spectra, e.g. the large or small values of $E_{i e}$ at each natural period corresponds well to Fourier's results. The effect of the duration $T_{s}$ on $E_{i e}$ can be seen very clearly, i. e. the value of $E_{i e}$ becomes larger as the duration $T_{s}$ becomes longer, when $(M \cdot D)$ and $G C$ are the same.

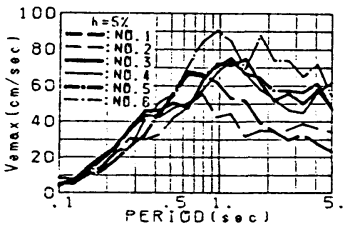

(a) Vemax

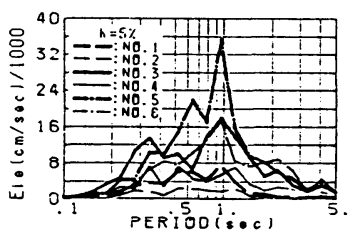

(b) Eie

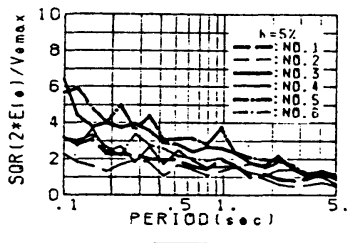

(c) $\sqrt{2 \mathrm{E} i \mathrm{e}} /$ Vemax

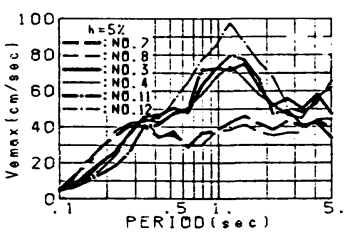

(a) Vemax

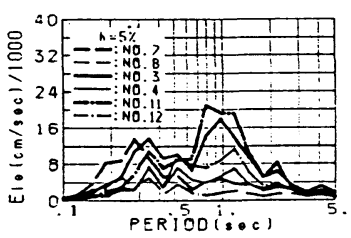

(b) Eie

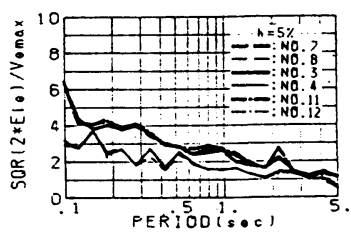

(c) $\sqrt{2 \mathrm{Eie}} /$ Vemax
Fig. 7 Effect of difference of $(M \cdot D)(G C=3, \quad(M \cdot D)=$ [A], [B], [C]) on $V_{e \max }, E_{l e}$ and $\sqrt{2 E_{t e}} / V_{\text {emax }}$.
Fig. 8 Effect of difference of $G C((M \cdot D)=[\mathrm{B}], G C=1$, $3,4)$ on $V_{e \max }, E_{t e}$ and $\sqrt{2 E_{i e}} / V_{\text {emax }}$. 
(c) The effect of the frequency characteristics of the input earthquake motion on $\sqrt{2 E_{t e}} / V_{\text {emax }}$ is reduced considerably, because the effects on both $V_{e \max }$ and $E_{l e}$, as was previously described in (a) and (b), cancel each other out. The results of all earthquakes show similar curves going down with the increase of the natural period $T$. However, the effect of the duration $T_{s}$ still remains, and the value of $\sqrt{2 E_{i e}} / V_{\text {emax }}$ increases as $T_{s}$ becomes longer.

Fig. 9 and Fig. 10 show the hysteretic energy $E_{h p}$ and the ratio of the equivalent velocity $\sqrt{2 E_{h p}}$ to the elastic maximum velocity $V_{e \max }\left(\sqrt{2 E_{h \rho}} / V_{e \max }\right)$, respectively, for the same earthquakes and damping factor as in Figs. 7 and 8.

Comparing these figures and (b) and (c) in Figs. 7 and 8, the following can be noted ;

(d) $E_{h \rho}$, which is one of the cumulative values of inelastic response, shows the tendency of the periodical characteristics which becomes smooth as the inelastic response becomes stronger according to the decrease of yield strength ratio $R$ (see Figs. 9 and 10). But the effect of the frequency characteristics and the duration of the input earthquake motion on this $E_{h p}$ is almost the same as the result on $E_{i e}$ as described in ( $b)$.

(e) The effect of the frequency characteristics of the earthquake on $\sqrt{2 E_{h \rho}} / V_{e \max }$ is almost the same as that on $\sqrt{2 E_{t e}} / V_{e \max }$, that is, the results of every earthquake show similar smooth curves going down according to the increase of the natural period $T$, and this ratio gets larger as the duration $T_{s}$ becomes longer, when $(M \cdot D)$ and $G C$ are the same. At this stage, the authors can not give any theoretical reason as to why the periodical characteristics of $\sqrt{2 E_{t e}} / V_{e \max }$ and $\sqrt{2 E_{h \rho}} / V_{e m a x}$ show the tendency to go down with the increase of $T$. However as can be seen in Figs. 7 to 10 , it seems to be caused by the relative difference of the periodical characteristics between $V_{e m a x}$ and $E_{i e}, E_{h p}$. Regarding this, Kato et al. ${ }^{1)}$ investigated the relation between the equivalent velocity $\sqrt{2 E_{d} / m}$ and $V_{e m a x}$, where $E_{d}$ is the input energy

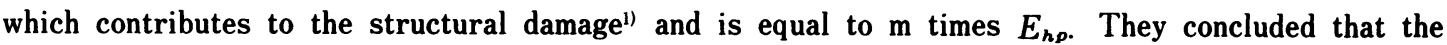
hypothesis proposed by Housner ${ }^{13)}, \sqrt{2 E_{d} / m}\left(=\sqrt{2 E_{h \rho}}\right)=V_{e \max }$, is approximately valid, without any regard to the structural parameters. However, the results in Figs. 9 and 10 show that Housner's hypothesis is a little unreasonable.

Summing up the results mentioned above, the maximum value of all responses at any time in the duration of an input earthquake motion is related to the natural period of the structure and is affected very much by the frequency characteristics of the earthquake. This is valid without any regard to elastic or inelastic response, as in the elastic maximum velocity $V_{e \max }$. On the other hand, as to the elastic input energy $E_{i e}$ and hysteretic energy $E_{h \rho}$, the value of the individual response which is accumulated at each interval is considerably affected not only by the frequency characteristics but also by the duration of the strong
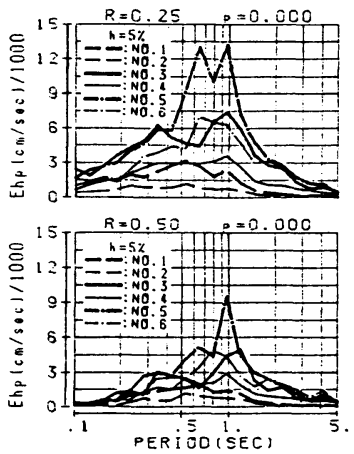

(a) Ehp
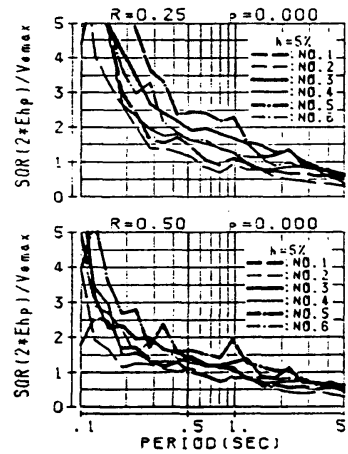

(b) $\sqrt{2 E h p} /$ Vemax

Fig. 9 Effect of difference of $(M \cdot D)(G C=3,(M \cdot D)=$ [A], [B], [C]) on $E_{h p}$ and $\sqrt{2 E_{h p}} / V_{e \max }$.
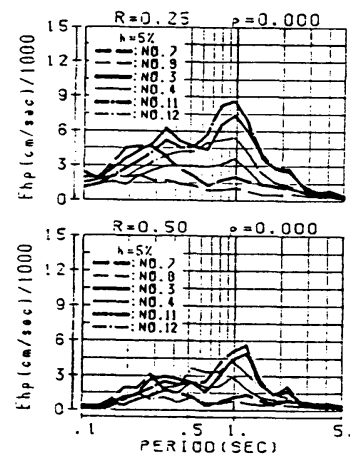

(a) Ehp
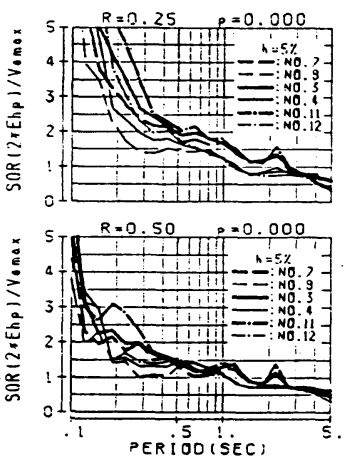

(b) $\sqrt{2 \mathrm{Ehp}} /$ Vemax

Fig. 10 Effect of difference of $G C\left((M \cdot D)=[\mathrm{B}], G_{c}=1\right.$, $2,3)$ on $E_{n p}$ and $\sqrt{2 E_{n p}} / V_{e \max }$. 
motion. Therefore, in the ratio of $\sqrt{2 E_{i e}}$ or $\sqrt{2 E_{h \rho}}$ to $V_{\text {emax }}$, the effect of the frequency characteristics on both ratios becomes very small, because they tend to cancel each other out. The effect of the duration $T_{s}$ on this ratio still remains.

\section{(3) Effect of the duration/the total power}

As was previously described, it is a major object of this study to evaluate the quantitative effect of the duration of input earthquake motions on the energy responses, such as $E_{i e}$ and $E_{h p}$.

In this section, we shall pay attention to the total power $P_{t}$ which has a close relation with the duration of the strong motion $T_{s}$ as shown in Table 1. Some consideration will be given to the comparison of $E_{i e}^{\prime}, E_{h p}^{\prime}$, $\sqrt{2 E_{i e}^{\prime}} / V_{e \max }$ and $\sqrt{2 E_{h p}^{\prime}} / V_{e \max }$ which are the normalized values of $E_{i e}, E_{h \rho}, \sqrt{2 E_{i e}} / V_{e \max }$ and $\sqrt{2 E_{h p}} / V_{e \max }$ by the standard total power $P_{t 0}$, respectively. The next formula is used to normalize these.

$$
E_{l}^{\prime}=E_{l} \cdot\left(P_{t 0} / P_{t I}\right)
$$

where, $E_{I}$ and $E_{I}^{\prime}$ are the actual value and the normalized value of energy response for the input earthquake $I^{\prime}$ and $P_{t I}$ and $P_{t 0}$ represent the total power of the earthquake $I$ and any total power used as the standard one, respectively.

Being normalized by the total power of the earthquake No. $5\left(P_{t 0}=P_{t 5}\right)$, the results of Figs. 7 and 9 are changed to Figs. 11 and 13. The results of Figs. 8 and 10 are also changed to Figs. 12 and 14 after being normalized by the total power of No. $11\left(P_{t 0}=P_{t 11}\right)$. In Figs. 13 and 14, only the results for the pair $(R=$ $0.25, p=0.0)$ are shown.

From the comparison of the normalized Figs. 11 to 14 with those of the original Figs. 7 to 10 , the following can be understood;

(a) The difference of the values in normalized $E_{i e}^{\prime}$ and $E_{h p}^{\prime}$ due to the duration $T_{s}$ for earthquakes with the same $(M \cdot D)$ and $G C$, become considerably smaller than that of their original $E_{i e}$ and $E_{h p}$, as can be seen from the comparison of the similar thick and thin lines in Figs. 11 to 14.

(b) When $G C$ is the same and $(M \cdot D)$ is different (see Figs. 11 and 13), the difference of the values of $E_{i e}^{\prime}$ and $E_{h p}^{\prime}$ due to $(M \cdot D)$ becomes smaller, except the range $T=0.6$ to 1 sec., where the response for $(M \cdot D)=[C]$ is dominant.

(c) When $(M \cdot D)$ is the same and GC is different (see Figs. 12 and 14), the effect of the frequency characteristics still remains and, even in the normalized $E_{t e}^{\prime}$ and $E_{h p}^{\prime}$, the difference of the value due to $G C$ cannot be canceled.

(d) As to $\sqrt{2 E_{i e}^{\prime}} / V_{\text {emax }}$ and $\sqrt{2 E_{h p}^{\prime}} / V_{\text {emax }}$, the above mentioned results given in (a) and (b) are
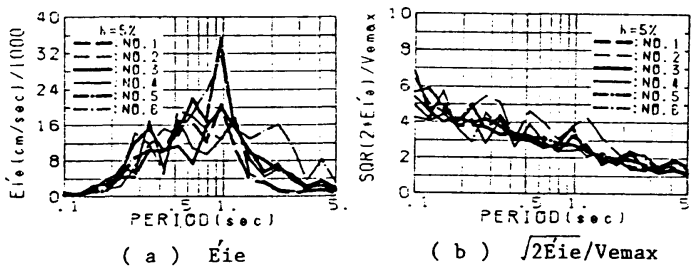

(b) $\sqrt{2 E i e} /$ Vemax

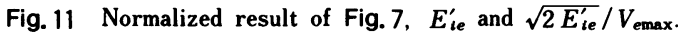
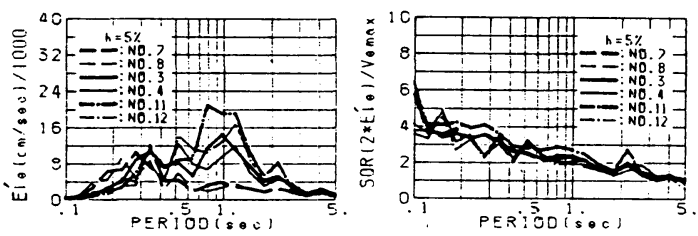

(a) Éie

(b) $\sqrt{2 E^{\prime} i e} /$ Vemax

Fig. 12 Normalized result of Fig. 8, $E_{t e}^{\prime}$ and $\sqrt{2 E_{t e}^{\prime}} / V_{e \max }$.

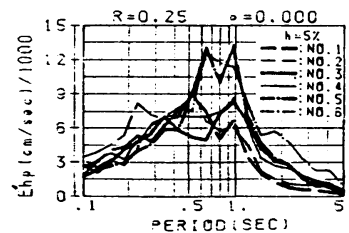

(a) Éhp

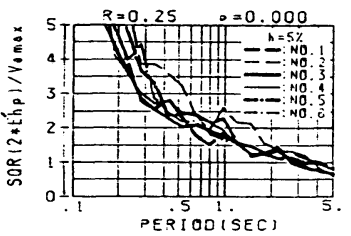

(b) $\sqrt{\text { 2Éhp } / \text { Vemax }}$

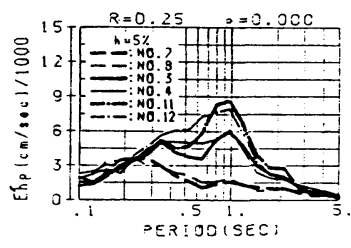

(a) Éhp

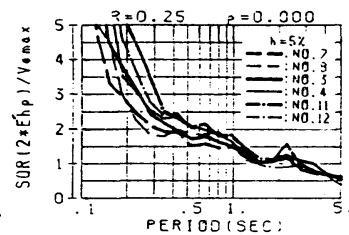

(b) $\sqrt{2 \text { Éhp }} /$ Vemax
Fig. 14 Normalized result of Fig. 10, $E_{h p}^{\prime}$ and $\sqrt{2 E_{h p}^{\prime}} / V_{e \max }$ 


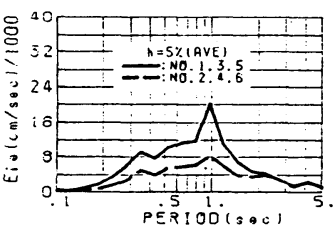

(a) Ele(ave) and Éie(ave)

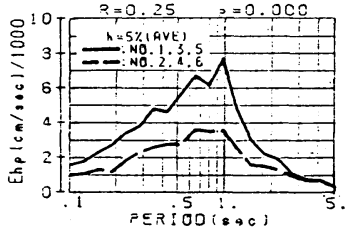

( b ) Ehp (ave) and Ehp (ave)

Fig. 15 Average response of $E_{i e}, E_{i e}^{\prime}$, and $E_{h p}, E_{h \rho}^{\prime}$ for No. $1,3,5$ and $2,4,6$.
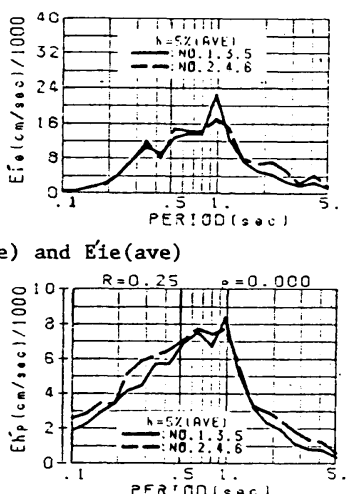

reflected, and the difference due to the input earthquake becomes smaller compared with the original ratio difference. From this result together with the result for $\sqrt{2 E_{h \rho}} / V_{e \max }$ described previously, we can possibly estimate the hysteretic energy $E_{h p}$, which represents a satisfactory index to evaluate the structural damage for severe earthquakes, through the structural parameters, the total power $P_{t}$ and the elastic maximum velocity $V_{\text {emax }}$ which are already familiar to us.

Though the analytical results were omitted, the effect of the input earthquakes on the inelastic input energy $E_{i p}\left(E_{i p}^{\prime}\right)$ is almost the same as that on $E_{i e}\left(E_{i e}^{\prime}\right)$ and $E_{h p}\left(E_{h p}^{\prime}\right)$.

As described above, for the input earthquakes with similar frequency characteristics, it seems that the total power $P_{t}$ can be an index to evaluate, the quantitative effect of the duration $T_{s}$ on the energy responses of the structures. Figs. 15 ( a ) and ( b ) show the comparison of the average value of $E_{i e}$ with $E_{i e}^{\prime}$ and $E_{h \rho}$ with $E_{h p}^{\prime}$, respectively, In these figures, the solid line shows the average value of the three earthquakes Nos. 1,3 and 5 which have the same ground condition $(G C=3)$, and the broken line shows that of Nos. 2, 4 and 6 which have a half of the duration $T_{s}$ for each earthquake Nos. 1,3 and 5 , respectively. In the case of $E_{i e}^{\prime}$ and $E_{h p}^{\prime}$ which are normalized by the total power of No. 3 , the difference of both averages is very small. This supports the validity of above mentioned theory.

Furthermore, as was described in section (2) of this chapter, the energy responses $E_{i e}$ and $E_{h p}$, whose responses are accumulated at any interval, are affected by the duration $T_{s}$ as well as the frequency characteristics of the input earthquake motion. This matter leads to the expectation that for the ratio of one form of energy to another, both effects are canceled out and the scattering of this ratio due to the difference in the input earthquake motions becomes smaller. This point is confirmed for the ratio of $E_{h p}$ to $E_{i e}$ in Fig. 16, if we exclude the range of the natural period $T$ less than 0.2 or 0.3 sec.

\section{CONCLUSIONS}

In this study, artificial earthquakes, each varying in frequency characteristics and duration, were generated so that they would comply with the acceleration response factor spectra shown in the Japanese specifications for road bridges $\mathrm{V}$. The numerical energy response analysis for bi-linear SDOF structures was also carried out by the use of these earthquakes. Then, based on the analytical results, the quantitative and qualitative effect of the frequency characteristics and duration of the input earthquake motions on the elastic maximum velocity $V_{e \max }$, the elastic input energy $E_{t e}$ and the hysteretic energy $E_{h p}$ were examined.

The results obtained in this study are summarized as follows;

(1) For those earthquakes whose acceleration response factor spectra are almost the same as the ones 
shown in the Japanese specifications for road bridges $V$, there is a near proportional relation between the duration of the strong motion $T_{s}$ and the total power $P_{t}$, when their maximum acceleration is the same.

(2) The maximum value of all responses, like the elastic maximum velocity $V_{e m a x}$, is related to the natural period of the structure and is greatly affected by the frequency characteristics of the input earthquake motion. On the other hand, as to the elastic input energy $E_{i e}$ and the hysteretic energy $E_{h \rho}$, the value of the responses which is accumulated at each interval is considerably affected not only by the frequency characteristics but also by the duration of the strong motion $T_{s}$.

(3) In connection with the result (2), for the ratio of the equivalent velocity $\sqrt{2 E_{t e}}$ or $\sqrt{2 E_{h p}}$ to $V_{e m a x}$, the effect of the frequency characteristics becomes very small, because the effect on both $E_{i e}$ or $E_{h \rho}$ and $V_{e m a x}$ is canceled out. Even in this ratio, however, the effect of the duration $T_{s}$ still remains. On the other hand, for the ratio of one form of energy to another such as $E_{h p} / E_{i e}$, both the effects are canceled out and the scattering of this ratio due to the difference of the input earthquake motions becomes smaller.

(4) In the case of earthquakes with similar frequency characteristics, a near proportional relation can be found between the value of the energy responses such as $E_{i e}$ and $E_{h p}$ of the structure and the total power $P_{t}$ of the input earthquake motion. Therefore, it seems that the total power $P_{t}$ can be seen as an index to evaluate the quantitative effect of the duration $T_{s}$ on the value of the energy responses.

These are the results obtained through limited numerical analysis, so that there still remain some problems to be solved, for example, what is the corresponding relation between the results for artificial earthquakes and the results for actual/recorded ones, how does one practically evaluate the quantitative effect of the duration $T_{s}$ on the energy responses and so on. These themes are worthy of more discussion in the future.

\section{REFERENCES}

1) Kato, B. and Akiyama, H. : Eenergy Input and Damages in Structures subjected to severe Earthquakes, Proc. of AIJ, No. 235 , pp. 9 18, September, 1975 (in Japanese).

2) Ohno, T., Nishioka, T. and Fujino, Y. : Quantitative Estimation of Plastic Energy absorbed in Structures subjected to Seismic Excitation, Proc. of JSCE, No. 333, pp.91 99, May, 1983 (in Japanese).

3) Kayano, S., Yamada, Y. and Iemure, H. : Seismic Design and Evaluation of Seismic Force by use of Input Energy Spectrum, The 39th Annual Meeting of JSCE, I -445, pp. 889 890, October, 1984 (in Japanese).

4) Hirao, K., Sawada, T. and Nariyuki, Y. : On the Hysteretic Energy Absorption in Structures under Strong Earthquake Motion, Proc. of EASEC-1, Vol. 3, pp. 1904 1915, January, 1986.

5) Hirao, K., Sawada, T., Nariyuki, Y. and Sasada, S. : On the Relation between Hysteretic Energy and Elastic Responses of Single Degree of Freedom Systems under Strong Earthquake Motions, Proc. of JSCE, No. 368/ I -5, pp. 401 410, April, 1986 (in Japanese).

6) Takeshima, T., Ohno, T. and Nishioka, T. : Linearized Estimation for Response of Elasto-Plastic Structures excited by Irregular Waves, Proc. of JSCE, No.344/ I -1, pp. 253 262, April, 1984 (in Japanese).

7) Hakuno, M. and Morikawa, O. : A Simulation on Important Factors to Collapse of Structures due to Earthquakes, Proc. of JSCE, No.344/ I-1, pp. 299 302, April, 1984 (in Japanese).

8) Zahrah, T.F. and Hall, W. J. : Earthquake Energy Absorption in SDOF Structures, Proc. of ASCE, Vol.110, No. ST 8, pp. 1757 1772, August, 1984.

9) Matsushima, M. and Matsui, K. : The Effect of Ground Motion upon Simple Structures, Proc. of Structural Engineering, Vol. 32 A, pp. 815 824, March, 1986 (in Japanese).

10) Japanese Road Association: Specifications for Road Bridges V, pp. 111 125, May 1980 (in Japanese).

11) Sawada, T. and Hirao, K. : A Generation Method of Artificial Earthquake complying with Target Response Spectrum, Proc. of the 17 th JSCE Conference on Earthquake Engineering, pp. 49 52, July, 1983 (in Japanese).

12) Sawada, T., Nagae, M. and Hirao, K. : A Definition for Duration of Earthquake Ground Motion by Phase Differences and its Statistical Analysis, Proc. of JSCE, No.368/ I -5, pp. 373 382, April, 1986 (in Japanese).

13) Housner, G. W. : Behavior of Structures During Earthquakes, Proc. of ASCE, Vol. 85, No. EM 4, pp. 109 129, October, 1959.

(Received August 27 1986) 